\title{
Target Detection Algorithm Based on Improved Gaussian Mixture Model
} Xiaomeng Wang ${ }^{1}$, Dequn Zhao ${ }^{1}$, Guangmin Sun ${ }^{1,2}$, Xingwang Liu ${ }^{1}$, Yanli Wu ${ }^{1}$

${ }^{1}$ Department of Electronic Engineering, Beijing University of Technology, Beijing, 100124, China

${ }^{2}$ Beijing Key Laboratory of Computational Intelligence and Intelligent System, Beijing, 100124, China

Email: gmsun@bjut.edu.cn

Keywords: Gaussian mixture model; Improved Gaussian mixture model; moving object detection.

\begin{abstract}
With the traditional Gaussian mixture model being more sensitive to light and failing to react to changes of lighting, a variances and a mean update program under local illumination and global illumination mutations are puts forward respectively in this paper. More specifically, a divergent method to update the mean of each Gaussian distribution in the background model is proposed, following the analyses of the average grey value of current image frame and the absolute difference of the average grey value of the background model. An innovative update method to update the variance of Gaussian mixture model is also presented, based on the study of the absolute value of the pixel value and mean value. Experimental results show that the algorithm can not only detect moving targets in a relatively more complete manner, but also exhibits better adaptability and robustness for outdoor lighting mutation.
\end{abstract}

\section{Introduction}

Detection of moving objects in image sequence is a fundamental step of information extraction in many vision applications such as visual surveillance, people tracking, traffic monitoring. Meanwhile, detecting moving objects is an essential step toward high-level image processing ${ }^{[1]}$.

Moving target detection methods are proposed mainly divided into three categories: optical flow method ${ }^{[2]}$, interframe difference method ${ }^{[3]}$ and background difference method $^{[4]}$. The optical flow method was proposed by Gibson in 1950, and optical flow method is a dense vector field. From analyzing of optical flow method, we can get abundant information about scenes and the moving targets ${ }^{[5]}$. But this algorithm is more complex and large amount of calculation, cannot satisfy the requirement of real-time processing of video streaming. Although the frame difference method has strong adaptability, but the timing of demand is higher, but depends on the velocity of the moving object. If the velocity of the object is faster, and the selected time interval is too big, it will cause no coverage between the two frames, making it impossible to segment the moving targets. If the velocity of the object is too slow, and the selected time is too small, it will cause excessive coverage, so that the detected object appears empty. Background difference method by using image sequence and the reference background model subtraction to detect moving targets, and can detect the moving targets related all pixels, but for the change of external environment (such as light, external events, etc.) are very sensitive ${ }^{[6]}$. In order to eliminate the influence of background disturbance of motion detection, a lot of background modeling algorithm were proposed. The most typical algorithm is a Gaussian mixture background model proposed by Stauffer and Grimson. Gaussian background modeling ${ }^{[7]}$ is modeling even to each pixel of the background, whose parameters will be updated with time increasing. However, this method cannot adapt to the light of mutation, which can lead to inaccurate target detection.

With the traditional Gaussian mixture model cannot adapt to the light mutation, an improved algorithm for the traditional Gaussian mixture model is proposed in this paper. The experiment result shows the method works better than the typical Gaussian mixture model. 


\section{Gaussian mixture model}

Gaussian background modeling is proposed by C.stauffer and WEL Grimson, the background is not only the sessions without movement, but the drifting leaves and waving watermark. Gaussian background modeling ${ }^{[8]}$ is modeling even to each pixel of the background, whose parameters will be updated with time increasing. The moving target is detected by whether the distribution of pixels of the target is coincident with Gaussian background modeling.

Establishment of mixture-of-Gaussian modeling.In this model, $\mathrm{K}(3<\mathrm{K}<5$ in general) pieces of Gaussian modeling will be established for each pixel. For the probability density function of the sampling value $x_{t}$ at time $\mathrm{T}$, it can be denoted by a sum of weighted probability density of $\mathrm{K}$ pieces of multi-dimensional Gaussian distribution function:

$$
P\left(x_{t}\right)=\sum_{i=t}^{K} W_{i, t} \eta_{i, t}\left(x_{t}, \mu_{i, t}, \Sigma_{i, t}\right)
$$

$\mathrm{K}$ is the number of Gaussian modeling. $W_{i, t}$ is the weight of the $\mathrm{i}^{\text {th }}$ Gaussian distribution at time t. $\mu_{i, t}$ is the mean value of the $\mathrm{i}^{\text {th }}$ Gaussian distribution at time t. $\Sigma_{i, t}$ is the matrix of co-variation of the $\mathrm{i}^{\text {th }}$ Gaussian distribution at time t. $\Sigma_{i, t}=\sigma_{i, t}^{2} I$ (For each pixel, $\sigma_{i, t}^{2}$ is the variation of the $\mathrm{i}^{\text {th }}$ Gaussian distribution at time $\mathrm{t}$ for the image sequence of each pixel), when each of the RGB observation component value of pixel is assumed as independent. $\eta_{i, t}\left(x_{t}, \mu_{i, t}, \Sigma_{i, t}\right)$ is the probability density function of the $\mathrm{i}^{\text {th }}$ Gaussian distribution at time t. $\eta_{i, t}\left(x_{t}, \mu_{i, t}, \Sigma_{i, t}\right)$ can be expressed as below. $\mathrm{n}$ is the dimension of $x_{t}$.

$$
\eta\left(x_{t}, \mu_{i, t}, \Sigma_{i, t}\right)=\frac{1}{(2 \pi)^{n / 2}\left|\Sigma_{i, t}\right|^{1 / 2}} \cdot \exp \left(-\frac{1}{2}\left(x_{t}-\mu_{i, t}\right)^{T} \sum_{i, t}^{-1}\left(x_{t}-\mu_{i, t}\right)\right)
$$

Model initialization. First, the value of $x_{t}($ when $\mathrm{t}=1)$ is treated as the mean value $\mu_{1}$ of the first Gaussian distribution, assigning $\sigma_{1}$ to a large value meanwhile. And then, we can make the detection by matching the sampling pixel value $x_{t}$ with the existed $\mathrm{k}$ pieces of Gaussian distribution. If we fail to match them, make a new Gaussian distribution to model when $k<K$, or make a new Gaussian distribution to substitute the one with smallest weight when $k=K$.

Parameters updation. We need to detect pixels first, and then match the pixel value $x_{t}$ of current frame to $\mathrm{k}$ pieces of Gaussian distribution in mixture-of-Gaussian model. It matches if expression (3) is satisfied. Then we can refer it into the model, and update the model regarding the new pixel value. The updated parameters of mixture-of-Gaussian model are shown as expression (4), (5),(6).

$$
\begin{aligned}
& \left|X_{T}-\mu_{t-1 . i}\right|<2.5 \sigma_{i} \\
& W_{i, t}=(1-\alpha) W_{i, t}+\alpha \\
& \mu_{i, t}=(1-\beta) \mu_{i, t-1}+\beta x_{t} \\
& \sigma_{i, t}^{2}=(1-\beta) \sigma_{i, t-1}^{2}+\beta\left(x_{t}-\mu_{i, t}\right)^{T}\left(x_{t}-\mu_{i, t}\right)
\end{aligned}
$$

The coefficient $\alpha(0 \leq \alpha \leq 1)$ represents the speed of background updating, which should be user-defined. The larger the value is, the faster the updating velocity is, and vice versa. $\beta$ is the rate of parameter learning. We need to establish a new Gaussian model and initialize parameters if the pixel value $x_{t}$ cannot match any of the Gaussian distributions. A larger and a smaller value should be picked up when $x_{t}$ is treated as mean, variation and weight accordingly. Finally the model which has the lowest probability in the original model will be substituted by the new Gaussian model. 
Target detection.In the background modeling, when there are one more Gaussian distribution relating to the pixel, the value of $\omega / \sigma$ will increase accordingly with the succeed-match time if we sort Gaussian distributions with a descending order of the value of $\omega / \sigma$. And then, we can determine the number of Gaussian distributions which can be used to express the background image by expression (7):

$$
B=\arg \min _{b}\left(\sum_{k=1}^{b} \omega_{k}>T\right)
$$

$\mathrm{T}$ represents the min weight ratio belonging to this background distribution. We commonly define the background with several Gaussian distribution when $\mathrm{T}$ is relatively large. If it is small, we can define the background with only one Gaussian distribution. The pixel value at time $t$ can be determined as expression (8):

$$
X_{b, t}=\sum_{i=1}^{B} \mu_{i, t} \times \omega_{i, t}
$$

The multi-model sessions can be better processed, when the establishment is modeling with multiple Gaussian distribution. Regarding this kind of background, the pixel value varies heavily at different time, while it should not be detected as foreground. Multiple independent color session is reasonable when we express the pixel value with multiple Gaussian distribution. During foreground detection, the pixel value belongs to background as long as the detected pixel value match any of the first B pieces of Gaussian distribution. If not, it will be treated as foreground.

\section{Improved Gaussian mixture model}

Variance Updating Method. We can achieve the result from expression (5), (6) that, background will be more stable if training time is longer. Meanwhile, the variation value $\sigma_{i, t}^{2}$ will be smaller due to the pixel value $x_{t}$ being closer to the mean value of Gaussian distribution. If variation is too small, the data which is the ones close to the mean value, will not be matched, and a low variation leads to a disadvantage of background model learning. Besides, we can also achieve the result from expression (3) that, several pixels which are not moving in the background will be treated as foreground if the estimated variation value is too small.

In this article, a new scheme is proposed about self-adaptive variance updating. First, we set a threshold value $T_{\sigma}$ used for variance updating, and then process the updating step in terms of two different situations. When $\left|X_{T}-\mu_{t-1 . i}\right|<T_{\sigma}$, it means the pixel value is close to the mean value. So we can introduce a slow-down factor $\kappa$ to expression in order to slow down the updating speed. If $\left|X_{T}-\mu_{t-1 . i}\right| \geq T_{\sigma}$, it means the pixel value is far from the mean value, and we can update the variation without any changes. The improved mixture-of-Gaussian modeling can express the variation value as below expression:

$$
\left\{\begin{array}{l}
\sigma_{i, t}^{2}=(1-\kappa \cdot \beta) \sigma_{i, t-1}^{2}+\kappa \cdot \beta\left(x_{i . t}-\mu_{i, t}\right)^{T}\left(x_{i . t}-\mu_{i, t}\right), \text { if }\left|x_{i . t}-\mu_{i, t}\right|<T_{\sigma} \\
\sigma_{i, t}^{2}=(1-\beta) \sigma_{i, t-1}^{2}+\beta\left(x_{i . t}-\mu_{i, t}\right)^{T}\left(x_{i . t}-\mu_{i, t}\right) \\
\text { else }
\end{array}\right.
$$

The slow-down factor has been assumed as $\kappa=x_{t}-\mu_{i, t} . \kappa$ will be smaller when the current pixel value is further from the mean value, and the variation value will be updated slower.

Mean Updating Method. The basic idea of this improvement is that, we can first determine the threshold $T_{\mu}$ of the average gray value $T_{\mu}$, and then calculate the absolute difference value between the average gray value of background model and the average gray value of the current image frame. If the absolute difference value is larger than $T_{\mu}$, a relatively large difference of light intensity is existed in the scene. So we need to update the mean values for each Gaussian distribution in the background model. If not, the mean value shall be updated as normal. Finally, the updating scheme is expressed as below: 
$\left\{\begin{array}{l}\mu_{i, t}=\mu_{i, t-1}+[\operatorname{mean}(t)-\operatorname{mean}(t-1)], \text { if }|\operatorname{mean}(t)-\operatorname{mean}(t-1)| \geq T_{\mu} \\ \mu_{i, t}=(1-\beta) \mu_{i, t-1}+\beta x_{t}, \text { else }\end{array}\right.$

$\operatorname{mean}(t)$ is the average gray value of the image frame at time $\mathrm{t}$.

Process of moving object detection algorithm.In this paper, the specific process of target detection method is shown in figure 1.

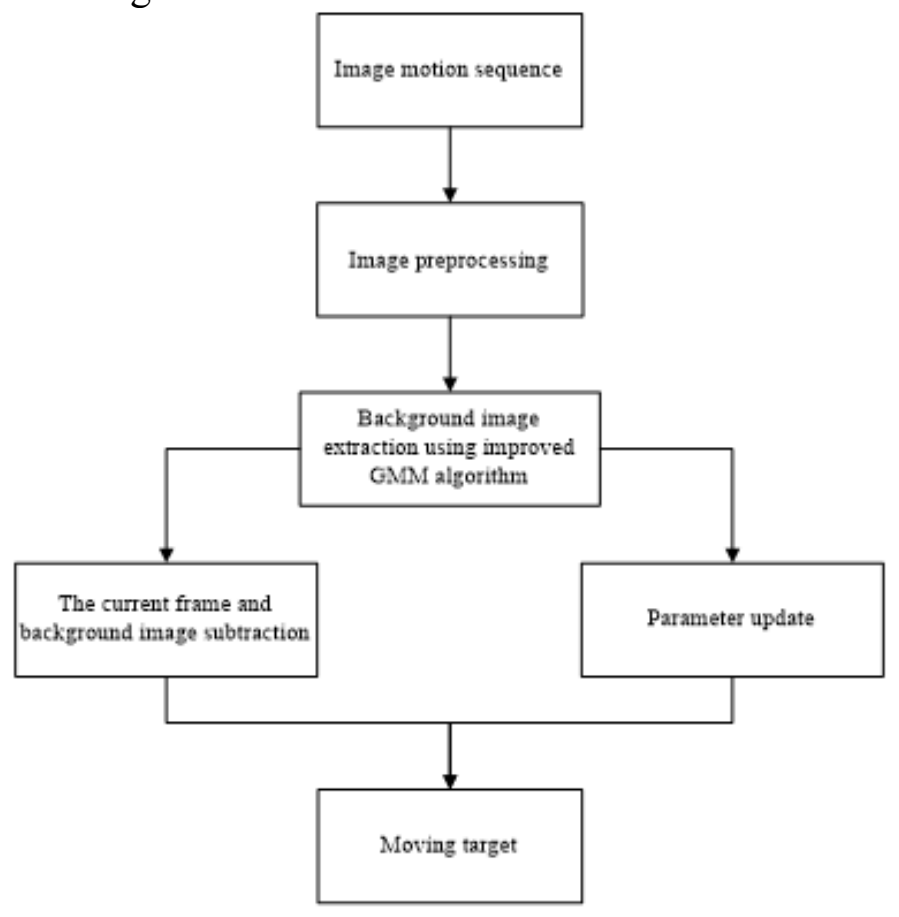

Fig.1 Process of moving object detection algorithm

\section{Experiment Results}

Based on Visual Studio 2008 and opencv2.0 software development tools, the system runs on a standard PC, processing speed is 15 frames per second,a resolution of $320 \times 240$.
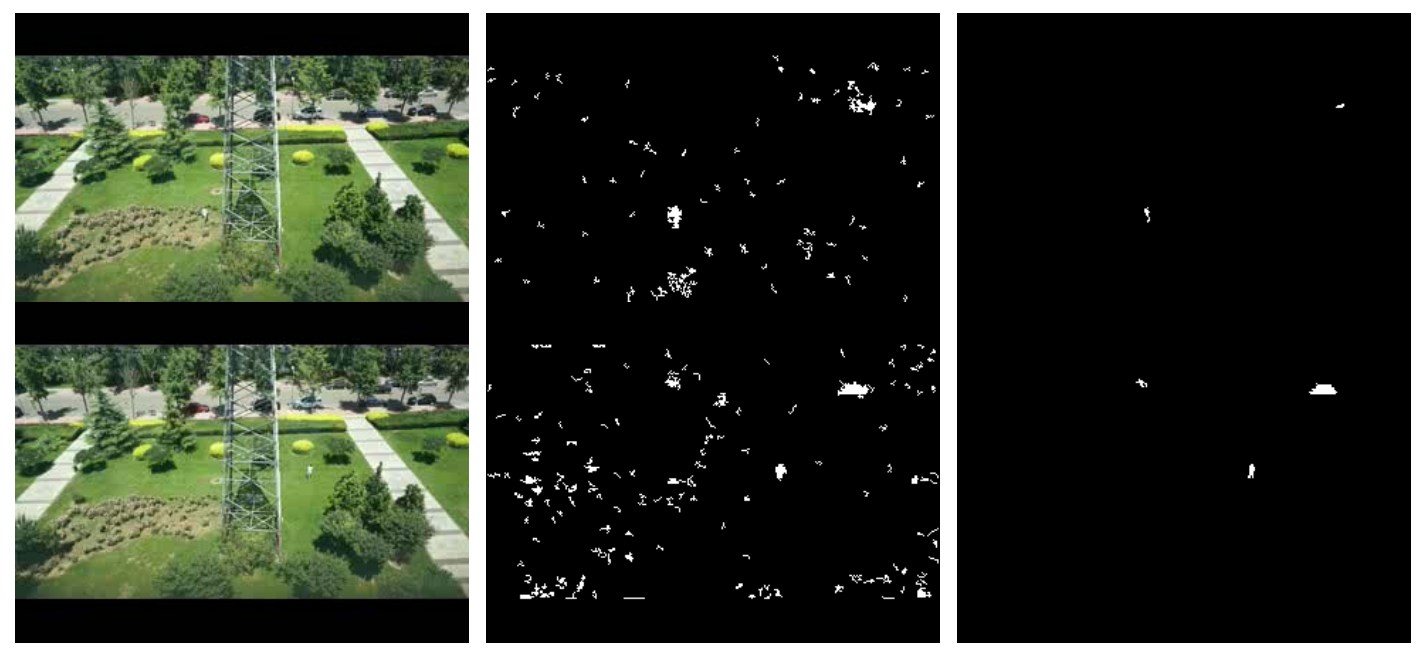

Fig.2 Results of two algorithms.

The figure 2 shows the experiment of improved algorithm comparing with the traditional Gaussian mixture model. The left column displays the original sequence at frames 279 and 3105 respectively. The middle column is the results of typical Gaussian mixture model for foreground images. The right column shows the foreground images adopting improved algorithm. Can be seen from the experimental results, a sudden change in the local and global illumination light, the 
traditional algorithm of mixed Gauss model is not very good, and cannot accurately detect moving targets. In the test results of the algorithm in this paper, the outline of moving targets with complete information, and there is no a large number of false target mistakenly identified, can adapt to illumination mutation, verify the validity of the improved algorithm in this paper.

\section{Summary}

Experimental results show that compared to the traditional Gaussian mixture background model algorithm, the improved method can effectively detect the moving targets. Even in cases of light mutation the generated background is relatively stable. The improved algorithm therefore demonstrates good adaptability to perform the task of real-time outdoor moving target detection and tracking.

\section{Acknowledgment}

This work has been supported by National Natural Science Foundation of China (20222201 and 61305026) and Beijing Municipal Commission of Education (KM200710005009, PXM2009_014204_09_000154 and KM201310005006).

\section{References}

[1] Bors A G, Pitas I. Prediction and t racking of moving object $s$ in image sequences [J].IEEE.T rans. on Image Processing , 2000,9 (8) :144121445.

[2] GAO Ping, SUN Xiangju, WANG Wei. Moving object detection based on Kiresh operator combined with optical flow[C]. Proceedings of IEEE Conference on Image Aanlysis and Signal, IEEE, 2010:622-624.

[3] XUE Lixia, LUO Yanli, WANG Zuocheng. Detection algorithm of adaptive moving objects based on frame difference method[J].Application Research of Computers, 2011, 28(4):1511-1559.

[4] YAN Rui,SONG Xue-hua, YAN Shu. Moving object detection based on an improved Gaussian mixture background model[C]//Proceedings of 2009 ISECS International Colloquium on Computing, Communication, Control, and Management. [S. 1.]: ISECS, 2009: 12-15.

[5] Barron J, Fleet D, Beauchemin S. Performance of optical flow techniques [J ] . I nternational J . Comp uter V ision ,1994,12 (1):42277.

[6] Valera M,Velastin S A. Inteillgent dist ributed surveillance systems : a review [J ] . I EE Proc. V ision. Image. Signal Process , 2005 ,152 (2) :1922204.

[7] STAUFFER C, GRIMSON W E L. Adaptive backgroud mixture models for real-time tracking[C]//Proceedings of IEEE Conference on Computer Vision and Pattern Recongnition. Fort Collins, Colorado: IEEE, 1999, 2: 246-252.

[8] JIAO BO, LI Guohui, TU Dan, et al. A fast convergent Gaussian mixture model for moving object detection[J]. Journal of Image and Graphics, 2008, 13(11): 2139-2143. 